\title{
Internal Facilitation Committees Improving Protocol Design Feasibility
}

\section{Kenneth A Getz* and Jennifer Kim}

Tufts Center for the Study of Drug Development, Tufts University, USA

\begin{abstract}
Biopharmaceutical companies are actively seeking ways to simplify study design complexity and improve protocol feasibility. One new approach adopted by a growing number of companies has been to establish internal facilitation committees charged with evaluating the benefits and costs of various study design elements (e.g. volunteer eligibility criteria and protocol procedures). In late 2012, Tufts CSDD conducted in-depth interviews and compiled and analyzed profiles of internal facilitation committees implemented recently by ten major pharmaceutical. This article discusses common committee characteristics and goals and highlights implementation challenges and early measures of committee impact.
\end{abstract}

Keywords: Protocol design; Protocol feasibility; Protocol complexity; Study design; Study feasibility

\section{Introduction}

Extensive research conducted by the Tufts Center for the Study of Drug Development (Tufts CSDD) during the past decade has captured and monitored a remarkable and troublesome trend: protocol designs supporting clinical trials have grown increasingly complex and ambitious, driving up drug study costs and adversely impacting clinical research performance [1].

Consider a typical phase III pivotal trial in 2012 versus that administered in 2002: The average number of endpoints per protocol has doubled; the median number of unique procedures and the total number of procedures performed per protocol has increased $48 \%$ and $57 \%$, respectively; the average number of volunteer eligibility criteria per protocol has nearly doubled; the average number of case report form pages supporting each protocol has tripled; and patients from an average of 34 countries and 196 research centers were recruited, up from 11 countries and 124 research centers [2].

Scholarly research suggests that the excessive collection of protocol data not only adds to study costs, but also indirectly and adversely affects the quality of more critical data elements and potentially harm data quality and analysis [3]. A recent Tufts CSDD study quantified the magnitude and cost of collecting protocol data that is not associated with primary or key secondary endpoints or regulatory requirements. Tufts CSDD analyzed 25, 103 procedures from 116 unique phase II and III protocols, and found that more than one out of every five procedures (22.3\%) support 'Non-Core' endpoints and objectives, (i.e., supplemental secondary, tertiary and exploratory), and that the direct cost to administer these 'Non-Core' procedures was $17.9 \%$ of an average clinical trial budget.

Scholarly research also shows that protocol complexity is associated with longer clinical trials and lower patient recruitment and retention rates $[4,5]$. To cite a few key findings published in the literature: health professionals are less likely to refer patients to clinical trials involving a large number of procedures [6]; more demanding protocols adversely affect study volunteer willingness to sign the informed consent form [7]; and complex protocols are also associated with higher levels of study volunteer dropout rates.

In response to high and rising levels of study design complexity, several years ago, a number of sponsor organizations began taking steps to establish internal facilitation committees. In late 2012, Tufts CSDD conducted in-depth interviews with ten of these organizations to profile committee goals, characteristics and implementation challenges.

\section{Methods}

In November 2012, Tufts CSDD contacted the 20 largest pharmaceutical and biotechnology companies to identify organizations that had implemented internal facilitation committees. Ten sponsor companies agreed to be profiled. Tufts CSDD then scheduled and conducted in-depth interviews that lasted approximately 45 minutes. Interviewees were executives at the senior director level or higher with direct involvement in establishing and managing these internal feasibility assessment mechanisms.

\section{Results}

Two of the ten companies profiled have had internal facilitation committees in place since 2009. Six pharmaceutical companies established their committees between 2010 and 2011. And two companies created their internal committees less than a year ago.

All of the companies have set up their committees as separate entities that report to senior management across several functional areas. Three of the ten committees report to clinical development; three report to clinical operations; one committee reports to medical affairs, and the remainder follow a matrix structure reporting to multiple functional areas.

Nearly all of the facilitation committees are comprised of crossfunctional representatives. Committees range in size from less than five members to more than ten, with the majority (seven out of the ten companies) having more than five members. Functional areas contributing personnel to the committees typically include clinical development; clinical operations; statistics; data management; medical writing; clinical pharmacology; regulatory; safety; and pharmacovigilance, in addition to finance and/or procurement.

Only one company reports having a dedicating full-time staff to its facilitation committee. The other nine recruit committee

*Corresponding author: Kenneth A Getz, Research Associate Professor, Director of Sponsored Research Programs, Tufts Center for the Study of Drug Development Tufts University, 75 Kneeland Street, Suite 1100, Boston, MA, USA 02111; E-mail: jjykim@gmail.com

Received July 27, 2013; Accepted September 05, 2013; Published September 09, 2013

Citation: Getz KA, Kim J (2013) Internal Facilitation Committees Improving Protocol Design Feasibility. Pharmaceut Reg Affairs 2: 111. doi:10.4172/21677689.1000111

Copyright: $\odot 2013$ Getz KA, et al. This is an open-access article distributed under the terms of the Creative Commons Attribution License, which permits unrestricted use, distribution, and reproduction in any medium, provided the original author and source are credited. 
members from a pool of cross-functional individuals who rotate on and off the committee, depending on capacity needs and required areas of expertise, as dictated by the protocols under review. In each committee, one or two members have responsibility for assigning committee members and capturing and disseminating information about committee progress and impact. At this time, only one of the ten committees offers their members formal training on assessing protocol feasibility.

Facilitation committees are typically charged with evaluating protocol feasibility, identifying areas to potentially lower protocol administration costs, and challenging specific procedures that are not associated with the primary and key secondary objectives and endpoints of the study.

Committee names vary widely between the ten profiled companies. To illustrate: One company calls it a 'Facilitated Clinical Review' committee; another the 'Protocol Financial Review' committee; and a third the 'Protocol Challenge' committee.

The missions and objectives of the internal facilitation committees across companies are similar, although some have a more expansive set of goals than do others. All of the committees are charged with simplifying and streamlining protocols by reducing unnecessary procedures. To meet this objective, as part of the review process, committees identify procedures not associated with primary and key secondary endpoints or regulatory requirements. Older committees have set additional objectives, including shortening study cycle times, lowering study costs and reducing the burden placed on study volunteers for their participation. To meet these objectives, committees assess the direct cost to perform extraneous procedures, as well as core procedures that may be conducted more frequently than necessary.

Committees typically provide input into the study design, just prior to final protocol approval. The program head typically submits a near-final version of the protocol to the facilitation committee. Recommendations for simplifying the study design are then returned to the program head, clinical teams and clinical operations. Acceptance and implementation of the recommendations are a function of the committee's level of visibility and support in the organization. Although a majority of committees report having some leverage to enforce their decisions (e.g. senior management support), in most cases, the decision to carry out committee recommendations ultimately rests with the program head and the clinical teams.

Internal facilitation committee oversight varies across the ten companies profiled. In several cases, the committees have broad review responsibility across all therapeutic areas and phases. In other companies, a narrower focus has been established due to time and capacity constraints, as well as portfolio priorities. One company performs facilitated feasibility review on all international protocols seeking more than 200 evaluable patients. In several companies, internal facilitation committees oversee only multi-specialty phase II and III protocols.

The committees use a variety of proprietary and commercial toolsincluding accounting, financial and protocol design applications-to compare their study design attributes to industry benchmarks, and to measure the direct cost of conducting protocol procedures.

Establishment and implementation of internal facilitation committees typically take 12 to 18 months, with substantial time devoted to overcoming internal areas of resistance (e.g. reluctance of staff to change long-established processes and the challenge of striking a closer balance between scientific and operating requirements), and to setting organizational expectations. As part of the implementation process, all of the companies report providing training for committee members to assist them in prioritizing their areas of focus and evaluation. During the early phases of implementation, most internal facilitation committees focus on a single, or a small number of studies, on a pilot basis.

All of the companies profiled plan to routinely collect metrics characterizing committee impact. These metrics are typically estimates of dollars or time saved, if committee recommendations are implemented. Many of the companies profiled have yet to collect enough impact data. Two of the older committees report that their internal facilitation committees have reduced the number of protocol procedures by $15-20 \%$; decreased the number of protocol amendments by $20-25 \%$; delivered fewer investigative sites that fail to enroll a single study volunteer; and shortened clinical trial cycle times by as much as $44 \%[8]$.

\section{Discussion}

The profiles summarized here represent a convenience sample of ten companies and the conclusions should be viewed with caution. Future research will benefit from a larger sample of companies and quantitative data to benchmark committee effectiveness and impact.

The establishment of internal facilitation committees signals a growing commitment among major pharmaceutical companies to adopt a more systematic and long-term approach to optimizing study design. Committees are positioned within their respective organizations as objective governance and assessment mechanisms, offering guidance and input into the existing protocol review process, without requiring organizations to alter legacy study design practices and procedures.

The committees facilitate a line of sight into study design, as it is being formulated and raise clinical team awareness of the impact that design decisions have on study budgets and on study execution feasibility. Committees routinely offer insight into how protocol designs can be streamlined and better 'fit to purpose'. Ultimately, internal facilitation committees may drive long-term change in study design practices.

The role and scope of internal facilitation committees will no doubt expand as more data is gathered that demonstrates their impact on cycle time and costs. The oldest established committees have already expanded their reach into more areas in the portfolio; they have more frequent interaction with clinical teams along the study design process, and they play a more active role in training and educating their organizations about the importance of feasibility in protocol design creation.

Internal facilitation committees are a promising new mechanism driving sustainable improvement in study design and protocol complexity and ultimately, delivering better clinical program performance, cost and quality.

\section{References}

1. Getz K, Campo R, Kaitin K (2011) Variability in protocol design complexity by phase and therapeutic area. Drug Information Journal 45: 413-420.

2. Getz KA, Stergiopoulos S, Marlborough M, Whitehill J, Curran M, et al. (2013) Quantifying the Magnitude and cost of collecting extraneous protocol data. Am $\mathrm{J}$ Ther.

3. Friedman L, Furberg C, DeMets D (2010) Data collection and quality contro in the fundamentals of clinical trials. Springer Science and Business Media 199-214. 
Citation: Getz KA, Kim J (2013) Internal Facilitation Committees Improving Protocol Design Feasibility. Pharmaceut Reg Affairs 2: 111. doi:10.4172/2167-7689.1000111

4. Getz KA, Wenger J, Campo R, Seguine E, Kaitin K (2008) Assessing the impact of protocol design change on clinical trial performance. Am J Therapeut 15: $450-457$

5. DiMasi JA, Grabowski HG (2012) R\&D costs and returns to new drug development: A review of the evidence: In The Oxford Handbook of the Economics of the Biopharmaceutical Industry.

6. Ross S, Grant A, Counsell C, Gillespie W, Russell I, et al. (1999) Barriers to participation in randomized controlled trials-A systematic review. J Clin Epidemiol 52: 1143-1156.

7. Madsen S, Holm S, Riis P (1999) Ethical aspects of clinical trials. Attitudes of public and out-patients. J Intern Med 245: 571-579.

8. Curran M (2012) Facilitated Clinical Sessions. Presentation at the optimizing study design and protocol development seminar, Philadelphia, PA, USA 